\title{
One-year results from the first US-based enhanced recovery after cardiac surgery (ERAS Cardiac) program
}

Judson B. Williams, MD, MHS, a,b Gina McConnell, RN, ${ }^{\mathrm{a}}$ J. Erin Allender, PharmD, ${ }^{\mathrm{a}}$ Patricia Woltz, PhD, RN, ${ }^{\mathrm{a}}$ Kathy Kane, MS, RN, ${ }^{\mathrm{a}}$ Peter K. Smith, MD, ${ }^{\mathrm{b}}$ Daniel T. Engelman, MD, ${ }^{\mathrm{c}}$ and William T. Bradford, MD

\section{ABSTRACT}

Objective: Our enhanced recovery after cardiac surgery (ERAS Cardiac) program is an evidence-based interdisciplinary process, which has not previously been systematically applied to cardiac surgery in the United States.

Methods: The Knowledge-to-Action Framework synthesized evidence-based enhanced recovery interventions and implementation of a designated ERAS Cardiac program. Standardized processes included (1) preoperative patient education, (2) carbohydrate loading 2 hours before general anesthesia, (3) multimodal opioid-sparing analgesia, (4) goal-directed perioperative insulin infusion, and (5) a rigorous bowel regimen. All cardiac anesthesiologists and surgeons agreed to follow the standardized pathway for adult cardiac surgery cases. The 1-year outcomes were compared between the 9 months pre- and post-ERAS Cardiac implementation using prospectively collected, retrospectively reviewed data.

Results: Comparing the pre- $(\mathrm{N}=489)$ with the post- $(\mathrm{N}=443)$ ERAS Cardiac groups, median postoperative length of stay was decreased from 7 to 6 days $(P<.01)$. Total intensive care unit hours were decreased from a mean of 43 to 28 hours $(P<.01)$. The incidence of gastrointestinal complications was $6.8 \%$ pre-ERAS versus $3.6 \%$ post-ERAS implementation $(P<.05)$. Opioid use was reduced by a mean of $8 \mathrm{mg}$ of morphine equivalents per patient in the first 24 hours postoperatively $(P<.01)$. Reintubation rate and intensive care unit readmission rate were reduced by $1.2 \%$ and $1.5 \%$, respectively ( $P=$ not significant $)$. The incidence of hyperglycemic episodes was no different after ERAS Cardiac initiation. Patient satisfaction was $86.3 \%$ pre-ERAS versus $91.8 \%$ post-ERAS Cardiac implementation and work culture domain scores revealed increases in satisfaction across all measured indices, including patient focus, culture, and engagement.

Conclusions: Initial clinical and survey data after the first year of a system-wide ERAS Cardiac program were associated with significantly improved perioperative outcomes. We believe this value-based approach to cardiac surgery can consistently result in earlier recovery, cost reductions, and increased patient/staff satisfaction. (J Thorac Cardiovasc Surg 2019;157:1881-8)

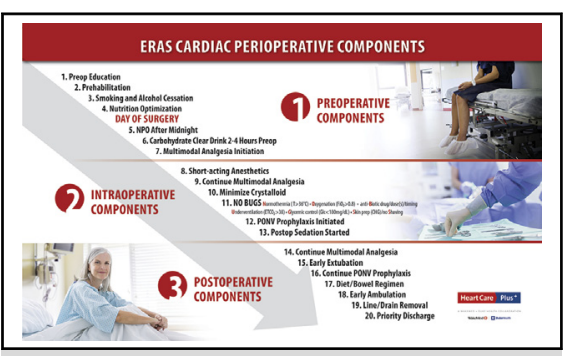

Flow graphic for ERAS Cardiac protocol.

Central Message

The study reports on systematic application of enhanced recovery principals to a US cardiac surgery program with reduced opioid use and LOS, and improved patient and staff satisfaction.

\section{Perspective}

Our ERAS Cardiac program is an evidencebased interdisciplinary process that has not previously been systematically applied to cardiac surgery in the United States. We believe this value-based approach to cardiac surgery can consistently result in earlier recovery, cost reductions, and increased patient/staff satisfaction.

See Commentary page 1889.

\footnotetext{
From the ${ }^{\mathrm{a}}$ WakeMed Health and Hospitals, Raleigh, NC; ${ }^{\mathrm{b}}$ Duke University School of Medicine, Durham, NC; and ${ }^{\mathrm{c} B a y s t a t e}$ Medical Center, Springfield, Mass.

J.B.W. and P.K.S. are supported in part by Grant U01-HL088953 from the National Institutes of Health Cardiothoracic Surgical Trials Network.

Read at the 98th Annual Meeting of The American Association for Thoracic Surgery, San Diego, California, April 28-May 1, 2018.

Received for publication April 27, 2018; revisions received Oct 25, 2018; accepted for publication Oct 28, 2018; available ahead of print Jan 18, 2019.

Address for reprints: Judson B. Williams, MD, MHS, WakeMed Clinical Research Institute, WakeMed Heart Center, and Duke University Department of Surgery, 300 New Bern Ave, Suite 1100, Raleigh, NC 27610 (E-mail: judson.williams@ duke.edu).

$0022-5223 / \$ 36.00$

Copyright (c) 2018 by The American Association for Thoracic Surgery

https://doi.org/10.1016/j.jtcvs.2018.10.164
}

Our enhanced recovery after cardiac surgery (ERAS Cardiac) program is an evidence-based interdisciplinary process that has not previously been systematically applied to cardiac surgery in the United States. In many surgical disciplines, specialty-specific enhanced recovery guidelines serve as a resource for individual institutions to implement highquality, evidence-based, perioperative care in standardized clinical pathways. $^{1-3}$ Feasibility studies have been

To view the AATS Annual Meeting Webcast, see the URL next to the webcast thumbnail. 


\section{Abbreviations and Acronyms \\ $\mathrm{CABG}=$ coronary artery bypass grafting \\ ERAS Cardiac $=$ enhanced recovery after cardiac surgery \\ GI = gastrointestinal \\ ICU $=$ intensive care unit \\ LOS $\quad=$ length of stay}

undertaken in cardiac surgery, but adoption of ERAS principles has been slow because of many barriers unique to the field of cardiac surgery. ${ }^{4,5}$ However, we hypothesized that an ERAS Cardiac program would reduce opioid use, gastrointestinal (GI) complications, and length of stay (LOS), and would improve patient and staff satisfaction. We present the testing of these hypotheses after 1 year of implementation of a designated ERAS Cardiac program.

\section{MATERIALS AND METHODS \\ Intervention}

The Knowledge-to-Action Framework synthesized enhanced recovery principles from other specialties to form an ERAS Cardiac protocol, the rationale, design, and implementation of which has been published. ${ }^{6}$ The Knowledge-to-Action Framework is composed of the 2 key components of knowledge creation and an action cycle. ${ }^{7}$ The action cycle outlines the activities, including selecting interventions and monitoring use, which are needed for knowledge to be applied in practice. Knowledge is adapted to the local context with assessment of local barriers and enablers, including involvement of all stakeholders in the care of the cardiac surgery patient. Figure 1 provides a graphic overview of the key components of the ERAS Cardiac protocol.

All cardiac surgeons, anesthesiologists, and intensivists agreed to apply the standardized protocol to all adult cardiac patients beginning on April 1, 2017. Key pathway interventions included (1) preoperative patient education, (2) carbohydrate loading 2 hours before general anesthesia $(355 \mathrm{~mL}$ including $22 \mathrm{~g}$ total carbohydrates), (3) age-adjusted multimodal opioidsparing analgesia, (4) goal-directed perioperative insulin infusion, and (5) a rigorous bowel regimen. ${ }^{6}$ Table 1 shows the details of the ERAS Cardiac interventions. There was no standardization of vasoactive infusion management or pacing wire or chest tube removal.

\section{Patients}

This study was approved by the institutional review board. All nonemergency adult cardiac surgery patients across a 919-bed community-based health system were included in the pre- and post-ERAS Cardiac protocol initiation. Percutaneous interventions such as transcatheter heart valve implantation were not included. The total study period was 18 months, including 9 months pre- and 9 months post-ERAS Cardiac initiation. There were no new procedures added during the study period.

\section{Outcomes}

Prospectively identified clinical outcomes were the following: length of hospital stay, length of intensive care unit (ICU) stay, time on ventilator, ICU readmission rate, reintubation rate, and GI complications. Prospective data collection included the predetermined clinical outcomes using standardized Society of Thoracic Surgeons database definitions, pharmacycollected opioid medication dispensation and administration, and patient satisfaction and workplace culture survey results. To reduce multiple outcomes bias and reporting bias in the study, the clinical outcomes to be measured were identified prospectively and limited to those hypothesized to be affected by the ERAS Cardiac pathway protocol.
GI complications were captured using the Society of Thoracic Surgeons Adult Cardiac Surgery Database definition: postoperative occurrence of any GI event, including but not limited to GI bleeding requiring transfusion, pancreatitis with abnormal amylase/lipase requiring nasogastric suction therapy, cholecystitis requiring cholecystectomy or drainage, mesenteric ischemia requiring exploration, prolonged ileus, or clostridium difficile. GI events may by definition require medical management, observational management, or surgical intervention to control. Prolonged ileus was defined as 3 days and may include nasogastric decompression, non per os status, and general surgery or gastroenterology consultation.

Opioid use was collected and reported as intravenous morphine equivalents use for the first 24 hours after surgery beginning with ICU arrival and not including intraoperative administration. Collection of opioid data involved a pharmacy-led audit of the electronic medical record for both dispensation of the medication and nursing medication administration.

Patient satisfaction was collected by positive or negative response to an established voluntary standardized survey instrument. ${ }^{8}$ The survey questioned hospital stay and was included with hospital discharge materials. Work culture survey domain scores were obtained for all full-time cardiothoracic ICU nursing staff members excluding nurse unit management. ${ }^{9}$

\section{Statistical Analysis}

Continuous variables were expressed as mean with standard deviation or as median with 25 th and 75 th percentiles. Categoric variables were expressed as frequencies and percentages with outcomes compared using Fisher exact test. Continuous outcomes were compared using Student $t$ test for means of normally distributed continuous variables and the Mann-Whitney $U$ nonparametric test for skewed distributions. All analyses were performed with SAS software (SAS Institute, Inc, Cary, NC).

\section{Post Hoc Analysis}

A limited post hoc analysis was undertaken to examine 2 outcomes not specified prospectively: incidence of postoperative hyperglycemia in the first 24 hours postoperatively and hospital inpatient readmission rate. Glycemic control was added surrounding concerns regarding the preoperative carbohydrate load, particularly in a patient population in whom diabetes mellitus is common. Hospital readmission rate after discharge was subsequently evaluated after reviewing the results on hospital LOS to determine whether readmission to the hospital was more common after introduction of the ERAS Cardiac pathway protocol.

\section{RESULTS}

\section{Study Population}

Included for the study were 489 patients in the 9 months before ERAS Cardiac go-live on April 3, 2017, and 443 patients for the first 9 months after initiation. Patient baseline characteristics are shown in Table 2. The principal operative case type was isolated coronary artery bypass grafting (CABG) in just more than $60 \%$ of cases. Mitral/tricuspid procedures and aortic procedures comprised $15 \%$ and $17 \%$ of cases, respectively.

The pre- and post-ERAS Cardiac cohorts were similar across all baseline characteristics, including age, sex, race, body mass index, congestive heart failure, prior stroke, chronic obstructive pulmonary disease, diabetes mellitus, and hemoglobin A1C. The presence of an intra-aortic balloon pump (16\%) and reoperation for bleeding $(1.5 \%)$ were also similar between the groups. 


\section{ERAS CARDIAC PERIOPERATIVE COMPONENTS}

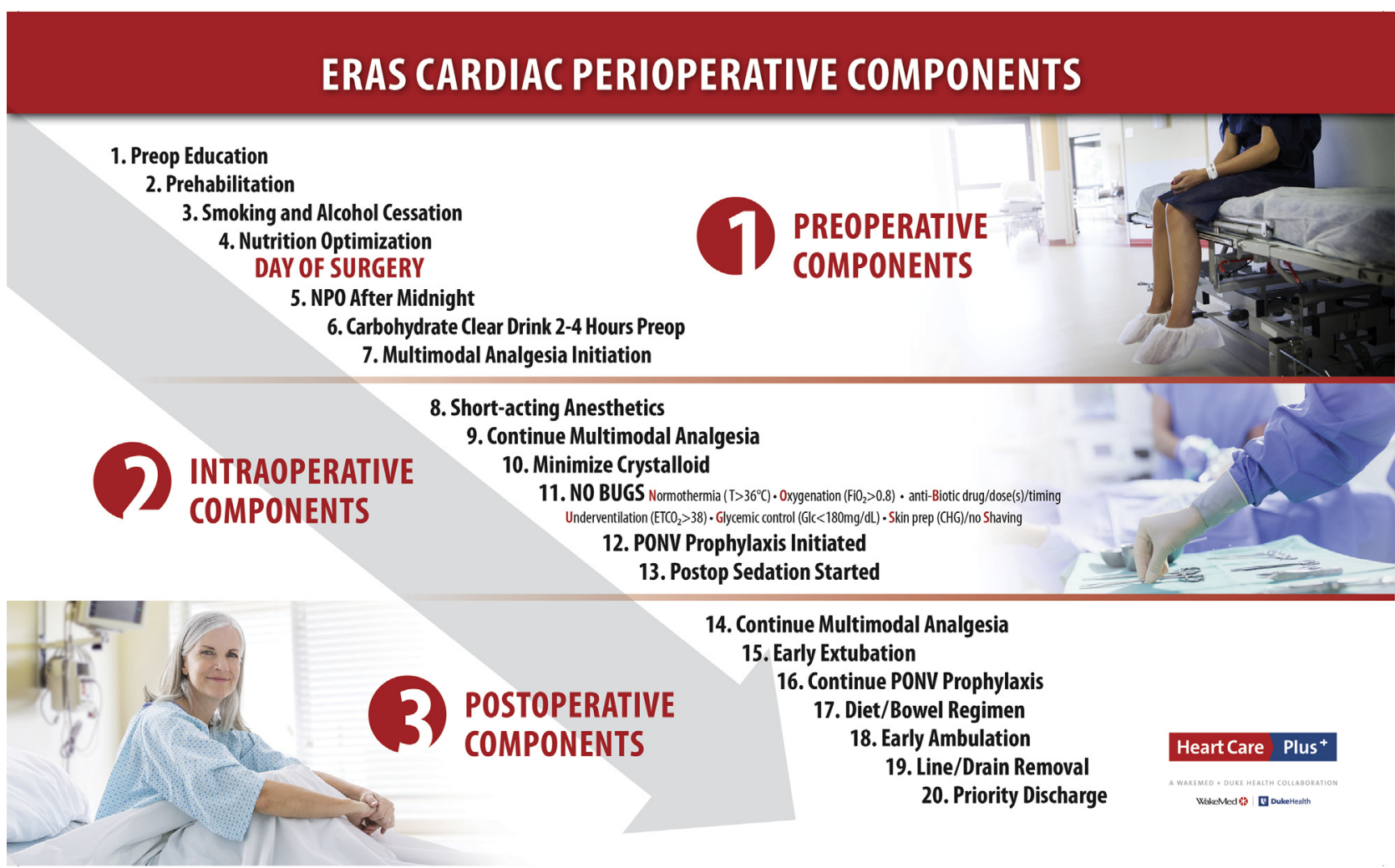

FIGURE 1. Graphic displaying the key components of the ERAS Cardiac protocol through each phase of perioperative care. $C H G$, Chlorhexidine gluconate; $E T C O 2$, end-tidal carbon dioxide; $N P O$, nil per os; $P O N V$, postoperative nausea/vomiting.

\section{Opioid Use}

In the study period before ERAS Cardiac implementation, the average intravenous milligrams of morphine equivalents used in the first 24 postoperative hours was 29 versus 21 in the post-ERAS Cardiac period. This approximately $30 \%$ reduction was statistically significant $(P<.01)$, as shown in Table 3.

\section{Clinical Outcomes}

LOS after surgery was reduced 1 day, median (25th, 75th) LOS $7(5,9)$ days pre- versus $6(5,8)$ post-ERAS Cardiac implementation $(P<.01)$. ICU LOS was also significantly reduced: median $(25$ th, 75 th) LOS $43(25,74)$ hours preversus $28(23,52)$ hours post-ERAS Cardiac implementation $(P<.01)$. ICU readmission rate was $5.1 \%$ pre versus $3.6 \%$ post $(P=$ not significant $)$, as shown in Table 3 .

Table 3 displays the results of postoperative ventilator times and reintubation rates, which were similar between the groups. GI complications were significantly reduced in the post-ERAS Cardiac period, with an approximately $50 \%$ reduction $(6.8 \%$ pre vs $3.6 \%$ post, $P<.05)$. Specific GI complications across both study cohorts were composed of prolonged postoperative ileus requiring medical therapy.
Median LOS among the patients having GI complications was 9 days in both the pre- and post-ERAS Cardiac study periods. Trends were similar among isolated CABG and non-CABG cases and among patients aged less than 65 years or 65 years and older. There were no appreciable differences when stratified by individual surgeon.

Post hoc analysis of the incidence of hyperglycemia (blood glucose $>180 \mathrm{mg} / \mathrm{dL}$ ) in the first 24 postoperative hours was no different between the groups $(18 \%$ pre vs $15 \%$ post). Post hoc analysis revealed the hospital inpatient readmission rate to be $8 \%$ pre- versus $9 \%$ post-ERAS Cardiac implementation, which shows no difference between the groups.

\section{Patient and Staff Satisfaction}

Figure 2 shows the results of domain scores for inpatient staff in the annual workplace culture survey. This consisted of anonymous responses of 38 cardiothoracic critical care nurses representing $100 \%$ of full-time staff and excluded participation by nurse management. Numeric improvements were seen across indices of patient focus, culture, and engagement in the most recent survey after ERAS Cardiac implementation, all improving from less than $76 \%$ positive response to greater than $83 \%$ positive 
TABLE 1. First-year protocol for enhanced recovery after cardiac surgery

\begin{tabular}{|c|c|}
\hline \multicolumn{2}{|l|}{ Consulting phase } \\
\hline Preoperative information, education, and counseling & $\begin{array}{l}\text { Patients and families receive dedicated preoperative counseling including information } \\
\text { packets }\end{array}$ \\
\hline Preoperative optimization & Medical optimization before surgery, including renal, pulmonary, and endocrine function \\
\hline Smoking and alcohol cessation & Smoking and alcohol cessation advised and promoted before surgery \\
\hline Preoperative nutritional status & $\begin{array}{l}\text { Patients screened for nutritional status and counseling given for a protein-rich diet at least } \\
\text { the week before surgery }\end{array}$ \\
\hline \multicolumn{2}{|l|}{ Preoperative phase } \\
\hline Preoperative fasting and carbohydrate treatment & $\begin{array}{l}\text { Patients are kept NPO } 8 \mathrm{~h} \text { before surgery except for an oral carbohydrate beverage given } \\
2-4 \mathrm{~h} \text { before surgery }\end{array}$ \\
\hline Preoperative multimodal analgesic initiated & Gabapentin $300 \mathrm{mg}$ and acetaminophen $1000 \mathrm{mg}$ given orally in preoperative holding area \\
\hline Anxiolytic medications & $\begin{array}{l}\text { Minimal anxiolytic medications given before surgery including midazolam given by } \\
\text { anesthesia provider }\end{array}$ \\
\hline \multicolumn{2}{|l|}{ Intraoperative phase } \\
\hline Intraoperative opioid administration & $\begin{array}{l}\text { Fentanyl IV given as needed for pain but typically }<1 \mathrm{mg} \text { for entire case } \\
\text { Hydromorphone } 0.5-1 \mathrm{mg} \text { given near completion of surgery }\end{array}$ \\
\hline Intraoperative multimodal analgesia & $\begin{array}{l}\text { If time since preoperative acetaminophen dose significantly exceeds } 6 \mathrm{~h}, 1 \text { dose of } \\
\text { acetaminophen } 1000 \mathrm{mg} \text { IV considered }\end{array}$ \\
\hline Postoperative sedation initiated & $\begin{array}{l}\text { Near end of surgery, dexmedetomidine or propofol infusion begun for postoperative } \\
\text { sedation in ICU while intubated }\end{array}$ \\
\hline \multicolumn{2}{|l|}{ Postoperative phase } \\
\hline Early extubation & $\begin{array}{l}\text { Patients extubated when medically appropriate with hemodynamic and pulmonary } \\
\text { stabilization. All sedation stopped upon decision made to wean patient from ventilatory } \\
\text { support for extubation }\end{array}$ \\
\hline Pulmonary function optimization & $\begin{array}{l}\text { Once extubated, patient expected to use incentive spirometry every } 2 \mathrm{~h} \text { while awake, have } \\
\text { head of bed elevated at least } 30 \text { degrees while sleeping, and out of bed to chair or } \\
\text { ambulating as much as tolerated while awake }\end{array}$ \\
\hline \multirow[t]{5}{*}{ Multimodal analgesia } & Acetaminophen $1000 \mathrm{mg}$ every $6 \mathrm{~h}$ \\
\hline & Gabapentin $300 \mathrm{mg}$ twice daily, ${ }^{*}$ weaned after POD 5 \\
\hline & $\begin{array}{l}\text { Oxycodone } 5-10 \mathrm{mg} \text { every } 4 \mathrm{~h} \text { as needed (liquid given through orogastric tube while } \\
\text { intubated, orally once extubated and tolerating clears) }\end{array}$ \\
\hline & Fentanyl IV for breakthrough pain resistant to oral medication management \\
\hline & $\begin{array}{l}\text { *Gabapentin } 100 \mathrm{mg} \text { given as maintenance dose if patient is aged }>70 \mathrm{y} \text { with hold } \\
\text { parameters for sedation }\end{array}$ \\
\hline \multirow[t]{2}{*}{ PONV prophylaxis } & Ondansetron $4 \mathrm{mg}$ IV every $4 \mathrm{~h}$ as needed \\
\hline & $\begin{array}{l}\text { Promethazine } 6.25-12.5 \mathrm{mg} \text { IV as needed for breakthrough nausea with avoidance if patient } \\
\text { aged }>70 \mathrm{y}\end{array}$ \\
\hline \multirow[t]{3}{*}{ Bowel motility } & Docusate $100 \mathrm{mg}$ orally each night starting POD 1 \\
\hline & Lactulose 20 -g solution orally POD 2 if no bowel movement \\
\hline & Bisacodyl $10 \mathrm{mg}$ suppository starting POD 1 , held for loose stools \\
\hline Glycemic control & $\begin{array}{l}\text { Target range for blood glucoses are } 110-150 \mathrm{mg} / \mathrm{d} \text {, with treatment algorithms standardized } \\
\text { for optimum glycemic control postoperatively }\end{array}$ \\
\hline Diet & $\begin{array}{l}\text { Ice chips allowed when extubated, diet advanced as tolerated after passing swallow } \\
\text { evaluation, gum chewing } 3 \text { times a day to promote bowel motility }\end{array}$ \\
\hline Early mobilization & $\begin{array}{l}\text { When hemodynamically stable and extubated, the patient is assisted out of bed to a chair } \\
\text { and activity advanced as tolerated to ambulation } 4 \text { times daily }\end{array}$ \\
\hline Lines and drain management & $\begin{array}{l}\text { Protocols in place for early removal of any orogastric/nasogastric tubes, central venous } \\
\text { access, invasive hemodynamic monitoring, urinary catheter, chest tubes, epicardial } \\
\text { pacing wires }\end{array}$ \\
\hline
\end{tabular}

$N P O$, Non per os; $I V$, intravenous; $I C U$, intensive care unit; $P O D$, postoperative day; $P O N V$, postoperative nausea and vomiting.

response $(P=.18)$. Patient satisfaction was $86.3 \%$ preversus $91.8 \%$ post-ERAS Cardiac implementation, an increase in positive responses by $5.5 \%$. These differences were not statistically significant.

\section{DISCUSSION}

We present the early results from an 18-month study of the first systematically applied US-based ERAS Cardiac program. We have found marked improvements in LOS, 
TABLE 2. Baseline characteristics of nonemergency adult cardiac surgery patients before and after enhanced recovery after cardiac surgery implementation

\begin{tabular}{lcc}
\hline \multirow{1}{*}{ Characteristics } & \multicolumn{2}{c}{ ERAS Cardiac } \\
\cline { 2 - 3 } & Pre- $(\mathbf{n}=\mathbf{4 8 9})$ & Post- $(\mathbf{n}=\mathbf{4 4 3})$ \\
\hline Age, mean, y & 65 & 65 \\
Female & 31 & 31 \\
\hline Nonwhite race & 26 & 29 \\
Body mass index & 29 & 29 \\
\hline Peripheral arterial occlusive disease & 11 & 13 \\
Congestive heart failure or left & 31 & 31 \\
$\quad$ ventricular ejection fraction $\leq 40 \%$ & & \\
\hline Prior stroke & 23 & 21 \\
Diabetes mellitus & 57 & 58 \\
Hemoglobin A1C & 6.5 & 6.5 \\
Operative case type & & \\
$\quad$ Isolated CABG & 62 & 61 \\
$\quad$ Mitral/tricuspid & 15 & 14 \\
Aortic & 16 & 18 \\
$\quad$ Other & 7 & 7 \\
\hline Intra-aortic balloon pump & 16 & 1.5 \\
Reoperation within first $48 \mathrm{~h}$ & 1.5 & \\
postoperative & & \\
\hline
\end{tabular}

Values are presented as percentages, unless otherwise indicated. ERAS Cardiac, Enhanced recovery after cardiac surgery; $C A B G$, coronary artery bypass grafting.

both ICU and total hospital LOS. We have also found significantly decreased incidence of postoperative GI complications and decreased opioid use. This idea of "enhanced recovery after surgery" was popularized by Henrik Kehlet in Denmark in the 1990s and spread in western Europe under the leadership of Olle Ljungqvist and colleagues. ${ }^{1}$ Initially designed for patients undergoing major abdominal operations, protocols are now well established for many types of surgical procedures, including GI, orthopedic, and gynecological. ${ }^{10-12}$
Although feasibility studies have been undertaken in cardiac surgery, adoption of enhanced recovery principles has been slower because of many barriers unique to the field of cardiac surgery. These barriers include strong individualized surgeon-based preferences, complex operations and systems of care, focus on traditional outcomes of mortality and stroke, and lack of published evidence for enhanced recovery protocols in cardiac surgery. Cardiac surgery is a specialty involving frequently lengthy operations, use of extracorporeal circulation, hypothermia, circulatory arrest, and hemodynamic manipulations, leading many to wonder what impact the changes of an ERAS Cardiac program would produce. Landmark studies in fast-track protocols, ${ }^{13}$ minimally invasive surgical approaches,${ }^{14}$ adoptions of evidence-based secondary prevention measures,${ }^{15}$ and clinical trial networks ${ }^{16}$ continue to advance the specialty with a robust evidence base. However, there exists a traditional focus on the technical aspects of the surgical procedures themselves with opportunities for study and innovation surrounding the perioperative care. ${ }^{11}$

We hypothesized that with the accumulation of marginal gains through the additive benefit of multiple positive small changes in each phase of care, we could ultimately achieve a major gain for our cardiac surgery patients. ${ }^{17}$ The weight of financial considerations is enormous in attempting to implement new protocols in today's environment, ${ }^{18}$ and as such, we proposed to health system leadership that investment in a systemwide implementation of ERAS Cardiac in our health system would result in cost savings and improvements in patient and staff satisfaction. The survey was a standardized annual survey for cardiothoracic ICU staff, a tremendously valuable human resource with high turnover at many centers. We believe that our nursing and ancillary staff felt more engaged and patientcentered, and had an improved understanding of the specific goals of care. The graphical display of the ERAS Cardiac process as shown in Figure 1 helps team

TABLE 3. Results for prespecified clinical outcomes among nonemergency cardiac surgery adult patients before and after enhanced recovery after cardiac surgery implementation

\begin{tabular}{|c|c|c|c|}
\hline \multirow[b]{2}{*}{ Clinical outcome } & \multicolumn{2}{|c|}{ ERAS Cardiac } & \multirow[b]{2}{*}{$P$ value } \\
\hline & Pre $(n=489)$ & Post $(n=443)$ & \\
\hline Opioid use in mean milligrams of intravenous morphine equivalents & 29 & 21 & $<.01$ \\
\hline Hospital LOS, median (25th, 75th), d & $7(5,9)$ & $6(5,8)$ & $<.01$ \\
\hline ICU LOS, median (25th, 75th), hours & $43(25,74)$ & $28(23,52)$ & $<.01$ \\
\hline Postoperative ventilator time, median (25th, 75 th), $\mathrm{h}$ & $5.2(3.9,7.3)$ & $5.3(3.9,6.9)$ & .53 \\
\hline Reintubation rate & 5.3 & 4.1 & .44 \\
\hline ICU readmission & 5.1 & 3.6 & .34 \\
\hline GI complications & 6.8 & 3.6 & .04 \\
\hline
\end{tabular}

Values are presented as percentages, unless otherwise indicated. $t$ tests were used for continuous variables other than opioid use, which used Mann-Whitney test. ERAS Cardiac, Enhanced recovery after cardiac surgery; $L O S$, length of stay; ICU, intensive care unit; GI, gastrointestinal. 


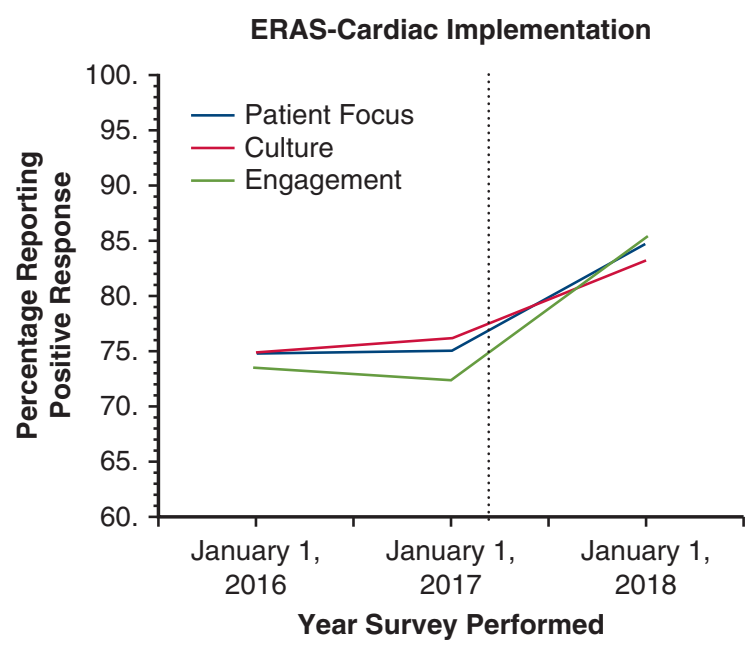

FIGURE 2. Workplace domain scores for inpatient staff before and after implementation of an ERAS Cardiac program. ERAS Cardiac, Enhanced recovery after cardiac surgery.

members understand how each component of care affects the others across each phase of the patient's care. A Hawthorne effect was likely important in early successes, and continuous quality improvement and education will be required to prevent early regression to a lower mean. Patient satisfaction was also increased with greater engagement after ERAS Cardiac implementation.

Our post hoc analysis of hyperglycemia was performed because of persistent concerns that the preoperative carbohydrate load might result in poor glucose control. In fact, the incidence of hyperglycemia was numerically reduced, potentially due to decreased insulin resistance. ${ }^{19}$ With such a large difference in postoperative LOS (1 day reduction), we examined hospital readmission seeking to examine whether or not our readmission rate was higher with earlier discharge, and it was not. Improvement in GI complications, primarily postoperative ileus, was expected with standardized bowel regimen and discontinuation of the preoperative fast. ${ }^{10}$ We report no difference in postoperative ventilator time and observed no individual case of aspiration in the setting of the carbohydrate load with ubiquitous use of transesophageal echocardiography and commonly encountered diabetic gastroparesis. ${ }^{20} \mathrm{~A}$ recently published Cochrane review examining 27 trials found similar results in noncardiac surgical patients. ${ }^{10}$

The observed reduction in opioid use was statistically significant with standardized multimodal analgesia. The reduction of early postoperative opioid use may have been contributory to the reduction in ICU LOS, which is often driven by issues surrounding sedation and pulmonary toilet after extubation. However, we only captured the first 24 postoperative hours and suspect that even more benefit was derived in days 2 to 4 after surgery, because we observed many patients not requiring any narcotic prescriptions upon hospital discharge. By only collecting pharmacy and nursing data for the first 24 hours postoperatively, we suspect that the impact of our ERAS Cardiac program was underestimated with regard to opioid use.

It is important to consider that the 9-month lead time (July 2016 to March 2017) for full implementation of the ERAS Cardiac program involved multiple working groups gathering input from all stakeholders including surgeons, anesthesiologists, intensivists, cardiologists, advance practice providers, patient advocates, clinical pharmacists, and nursing staff from preoperative, intraoperative, and postoperative phases of care. It required many hours of education of staff on inpatient and outpatient units. The process of program design and implementation likely affected patient care before the actual ERAS Cardiac go-live date, and therefore the 9 months of data capture before April 2017 is likely representative of a cohort benefiting from the education and early adoption of many of our ERAS principles. It follows that bias in this study would be toward the null hypothesis, strengthening confidence in our positive findings, as opposed to comparing our present results with a historical control cohort from a past year.

Future work for the next year of our ERAS Cardiac program will include a focus on a delirium prevention bundle, oral hygiene, perfusion management, and goal-directed care. We are also building into our electronic medical record a pathway for collecting nontraditional outcomes, including time to first ambulation, opioid prescriptions at hospital discharge, and the time until return to work, place of worship, or school. Because audit is paramount to a successful continuous quality improvement platform, implementation of an automated ERAS audit system is a future goal to allow focus on areas where pathway compliance is lower and to allow teams to iterate toward improved adherence and improved outcomes. ${ }^{21}$

\section{Study Limitations}

The present study represents a natural experiment in the application of an ERAS Cardiac program system-wide without other programmatic changes including surgeons. Because the present study is nonrandomized, measured or unmeasured confounders could have influenced our findings. To limit the multiple-outcomes bias common in observational studies, the outcome measures presented were prospectively identified for study based on review of the ERAS outcomes literature applied to other specialties.

\section{CONCLUSIONS}

Initial clinical data after the first year of a hospital-wide ERAS Cardiac program were associated with significantly improved perioperative outcomes. We believe this valuebased approach to cardiac surgery can result in earlier 
recovery; reduced opioid use, LOS, and cost; and increased patient/staff satisfaction.

\section{Webcast}

You can watch a Webcast of this AATS meeting presentation by going to: https://aats.blob.core.windows.net/ media/18Apr30/28DE\%20Perioperative \%20Care/S73_1_ webcast_020305710.mp4.

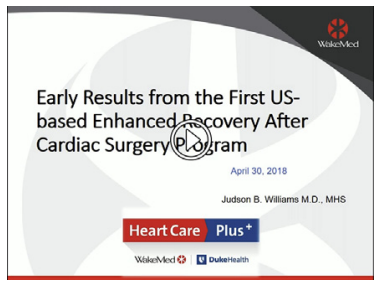

\section{Conflict of Interest Statement}

D.T.E.: Consulting for Astute Medical, Zimmer-Biomet, and Edwards Lifesciences. All other authors have nothing to disclose with regard to commercial support.

\section{References}

1. Ljungqvist $\mathrm{O}$, Scott M, Fearon KC. Enhanced recovery after surgery: a review. JAMA Surg. 2017;152:292-8.

2. Thiele RH, Rea KM, Turrentine FE, Friel CM, Hassinger TE, McMurry TL, et al. Standardization of care: impact of an enhanced recovery protocol on length of stay, complications, and direct costs after colorectal surgery. J Am Coll Surg. 2015;220:430-43.

3. Kim MP, Chan EY, Meisenbach LM, Dumitru R, Brown JK, Masud FN. Enhanced recovery after thoracic surgery reduces discharge on highly dependent narcotics. J Thorac Dis. 2018;10:984-90.

4. Fleming IO, Garratt C, Guha R, Desai J, Chaubey S, Wang Y, et al. Aggregation of marginal gains in cardiac surgery: feasibility of a perioperative care bundle for Enhanced Recovery in Cardiac Surgical Patients. J Cardiothorac Vasc Anesth. 2016;30:665-70.

5. Krzych $€$, Kucewicz-Czech E. It is time for enhanced recovery after surgery in cardiac surgery. Kardiol Pol. 2017;75:415-20.

6. McConnell G, Woltz P, Allender JE, Bradford WT, Williams JB. Design, rationale, and implementation of an enhanced recovery after cardiac surgery program. Nursing. October 3, 2018 [Epub ahead of print].

7. Graham I, Logan J, Harrison M, Straus S, Tetroe J, Caswell W, et al. Lost in knowledge translation: time for a map? J Contin Educ Health Prof. 2006;26: 13-24.

8. Hospital Consumer Assessment of Healthcare Providers and Systems. Centers for Medicare \& Medicaid Services, Baltimore, MD. Available at: http://www. hcahpsonline.org. Accessed April 20, 2018.

9. Nursing Engagement Survey. Washington, DC. Available at: https://www. advisory.com/talent-development/employee-engagement-initiative. Accessed April 20, 2018.

10. Smith MD, McCall J, Plank L, Herbison GP, Soop M, Nygren J. Preoperative carbohydrate treatment for enhancing recovery after elective surgery. Cochrane Database Syst Rev. 2014;8:CD009161.

11. Li M, Zhang J, Gan TJ, Qin G, Wang L, Zhu M, et al. Enhanced recovery after surgery pathway for patients undergoing cardiac surgery: a randomized clinical trial. Eur J Cardiothorac Surg. 2018;54:491-7.

12. Enhanced Recovery after Cardiac Surgery Society. Available at: www. erascardiac.org/. Accessed August 12, 2018.

13. Engelman RM, Rousou JA, Flack JE III, Deaton DW, Humphrey CB, Ellison LH, et al. Fast-track recovery of the coronary bypass patient. Ann Thorac Surg. 1994; 58:1742-6.

14. Williams JB, Peterson ED, Brennan JM, Sedrakyan A, Tavris D, Alexander JH, et al. Association between endoscopic vs open vein-graft harvesting and mortal- ity, wound complications, and cardiovascular events in patients undergoing CABG surgery. JAMA. 2012;308:475-84.

15. Williams JB, Delong ER, Peterson ED, Dokholyan RS, Ou FS, Ferguson TB Jr, Society of Thoracic Surgeons and the National Cardiac Database. Secondary prevention after coronary artery bypass graft surgery: findings of a national randomized controlled trial and sustained society-led incorporation into practice. Circulation. 2011;123:39-45.

16. Smith PK, Michler RE, Woo YJ, Alexander JH, Puskas JD, D’Alessandro DA, et al. Design, rationale, and initiation of the surgical interventions for moderate ischemic mitral regurgitation trial: a report from the cardiothoracic surgical trials network. J Thorac Cardiovasc Surg. 2012;143:111-7.e1.

17. Anselmi A, Flecher E. Minor postoperative morbidity should not be neglected. $J$ Thorac Cardiovasc Surg. 2016;152:602.

18. Engelman DT. Surgical economics: MACRA, MIPS, and bundles-Lessons learned in the first 3 years of a coronary artery bypass grafting alternative payment model. J Thorac Cardiovasc Surg. 2017;153:381-4.

19. Noblett SE, Watson DS, Huong H, Davison B, Hainsworth PJ, Horgan AF. Preoperative oral carbohydrate loading in colorectal surgery: a randomized controlled trial. Colorectal Dis. 2006;8:563-9.

20. Williams JB, Peterson ED, Albrecht ÁS, Li S, Hirji SA, Ferguson T Jr, et al. Glycemic control in patients undergoing coronary artery bypass graft surgery: clinical features, predictors, and outcomes. J Crit Care. 2017;42: 328-33.

21. Nelson G, Dowdy SC, Lasala J, Mena G, Bakkum-Gamez J, Meyer LA et al. Enhanced recovery after surgery $(E R A S \circledast)$ in gynecologic oncology practical considerations for program development. Gynecol Oncol. 2017;147 617-20.

Key Words: cardiac surgery, enhanced recovery, perioperative care, value, outcomes, complications

\section{Discussion}

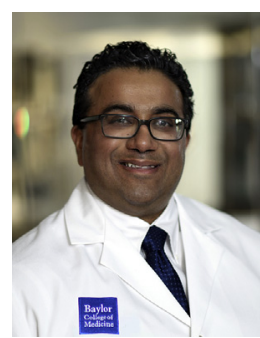

Dr S. Chatterjee (Houston, Tex). Dr Williams and colleagues at Wake Med and Duke should be congratulated for implementing and demonstrating the efficacy of an ERAS program within the United States in cardiac surgery. I have 4 specific questions.

The Hawthorne effect basically says that when you know you are being observed, people tend to behave better, tend to be able to follow through with outcomes, and I am curious how much of a Hawthorne effect might there have been in ICU LOS? Specifically, what you have demonstrated is that there is a 15 -hour reduction in median length of ICU stay. Nevertheless, the time to extubation did not change; it was 5 hours in both groups. You had a pretty sick cohort of patients: $16 \%$ of patients had balloon pumps compared with the Society of Thoracic Surgeons average, which is approximately $8 \% ; 20 \%$ of patients had prior strokes. So I am wondering, did you do anything else during this time period since the ventilator time stayed the same? Did you take the balloon pumps out sooner, did you wean the drips off faster, things that might be part of a fasttrack protocol, because if those things stayed the same, how do you explain the reduction in ICU LOS? 


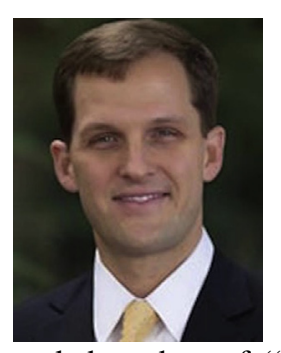

Dr Williams (Raleigh, NC). I think you hit the nail on the head. There was a Hawthorne effect, and that's one idea behind enhanced recovery platforms. It's an idea, it's a movement, it's a platform for accumulation of marginal gains, and one of those marginal gains is the engagements of our staff and the ethos of "we know what the plan is." We don't have 7 different preference lists for 7 different cardiac surgeons in our hospital anymore: We have 1 protocol, and that speeds everything up. Will we observe in the next year a regression toward the mean? Most likely. All good interventions do; there is typically a regression toward the mean. But I believe much of this work is sustainable.

Dr Chatterjee. Did you notice certain subsets of patients who benefited more or less, specifically, coronaries versus valves, say older patients versus young patients, diabetic versus nondiabetic patients, or higher Society of Thoracic Surgeons risk, maybe like 3 or 4, compared with lower Society of Thoracic Surgeons risk?

Dr Williams. We have not done any subgroup analyses. We kept this as pure and PICO methodology directed as possible. This was not throw 5000 patients into a database and let's look at every possible outcome and see what we find. This was true prospectively identified outcomes in a regimented way.

One thing I am interested in looking at is those who we excluded, because the emergency cases still benefited from the postoperative care. That would be another interesting subgroup to examine.

Dr Chatterjee. Your article alludes to this and you mentioned this briefly. You looked at opioid use in the first 24 hours, glucose management in the first 24 hours. Do you have data on how things went for the rest of the hospital course?

Dr Williams. I wish we had captured opioid use for the duration of the hospital stay, even for the first several weeks postoperatively, because I believe the greatest reductions that were seen in opioid use were actually more like postoperative day 3,4 , we had patients going home with no narcotic prescription, whereas 3 or 4 years ago in our health system everyone was going home with Percocet and now patients are going home with no narcotics at all. Again, I think that only strengthens our conclusions, because that is a weakness in the study, but it biases toward the null hypothesis, which is no difference, and the fact that we showed a difference even just in those first 24 hours is huge. But that's one thing in particular I want to look at going forward. We want to reduce opioids for our inpatients and opioids out in the community.

The glucose control was a post hoc analysis, and the reason for that is every time we speak about ERAS and these ideas, that's a major concern with our carbohydrate load, well, you are going to have spikes and hyperglycemia, and actually numerically hyperglycemia went down, and I believe it's because of insulin resistance. And the similar trends that other surgical specialties have observed, they may actually improve perioperative glycemic control.

Dr Chatterjee. How generalizable do you think, based upon this experience, is this to 2 unique sets of patients: one is patients in more socioeconomically disadvantaged groups who tend to be taken care of at public hospitals, and second, patients in whom there is a longer distance referral practice where the patients are coming from farther out, especially with respect to not changing the readmission rate without sacrificing that?

Dr Williams. We believe this is reproducible at least for these types of cases. So these include on-pump and offpump coronaries, valves, minimally invasive, full sternotomy, circulatory arrest cases, complex aortic work, redo surgery, and so forth. We think for the scope of adult cardiac surgery, this is relevant. How is it going to apply to transplantation, for example? This requires a working group, and we have to look at how medications interact with the transplant meds and that sort of thing. It remains to be seen how effective we can be at reproducing this. Although our main campus is a 919-bed facility, I think this would be even easier to roll out at smaller facilities where less staff at each phase of care has to be educated. 